Chromosome Res., in press. 28-6-2017

Published version on: http://www.springerlink.com/content/0967-3849

The final publication is available at Springer via https://link.springer.com/article/10.1007/s10577-017-9560-1

\title{
Genomic properties of chromosomal bands are linked to evolutionary rearrangements and new centromere formation in primates
}

Concetta Federico $^{1 *}$, Anna Maria Pappalardo ${ }^{1}$, Venera Ferrito ${ }^{1}$, Sabrina Tosi $^{2}$,

Salvatore Saccone ${ }^{1}$

${ }^{1}$ Dept. Biological, Geological and Environmental Sciences, Section of Animal Biology, University of Catania, Italy.

${ }^{2}$ Centre for Cell and Chromosome Biology, Division of Biosciences, Brunel University, West London, United Kingdom.

* Corresponding author: Concetta Federico, PhD

Dept. Biological, Geological and Environmental Sciences

Section of Animal Biology "M. La Greca",

Via Androne, 81

95124 Catania

Italy

Tel +39.095.7306037-38

E-mail: federico@unict.it 


\begin{abstract}
Chromosomal rearrangements in humans are largely related to pathological conditions, and phenotypic effects are also linked to alterations in the expression profile following nuclear relocation of genes between functionally different compartments, generally occupying the periphery or the inner part of the cell nuclei. On the other hand, during evolution chromosomal rearrangements may occur apparently without damaging phenotypic effects, and are visible in currently phylogenetically-related species. To increase our insight into chromosomal reorganisation in the cell nucleus, we analysed eighteen chromosomal regions endowed with different genomic properties in cell lines derived from eight primate species covering the entire evolutionary tree. We show that homologous loci, in spite of their evolutionary relocation along the chromosomes, generally remain localised to the same functional compartment of the cell nuclei. We conclude that evolutionarily succesfull chromosomal rearrangements are those that leave the nuclear position of the regions involved unchanged. On the contrary, in pathological situations, the effect typically observed is on gene structure alteration or gene nuclear reposition. Moreover, our data indicate that new centromere formation could potentially occur everywhere in the chromosomes, but only those emerging in very GC-poor/gene-poor regions, generally located in the nuclear periphery, have a high probability of being retained through evolution. This suggests that, in the cell nucleus of related species, evolutionary chromosomal reshufflings or new centromere formation does not alter the functionality of the regions involved or the interactions between different loci, thus preserving the expression pattern of orthologous genes.
\end{abstract}

Key words: $\quad$ human genome; primate chromosomes; interphase nuclei; BAC probes; new centromeres; evolutionary chromosomal rearrangements 


\section{Introduction}

The karyotypes of the extant primate species are the result of a series of chromosomal rearrangements that have occurred during evolution. The current hypotheses regarding the ancestral primate karyotype and the identification of evolutionary chromosomal rearrangements, which have led to the karyotypes of the current species, derive from a large number of studies beginning with the analysis of chromosomal banding (see Seuánez 1979 for a review). These were followed by more detailed studies using molecular cytogenetic techniques, such as fluorescence in situ hybridisation (FISH) with cross-species chromosome painting (Muller et al. 1999) and with bacterial artificial chromosomes (BACs) containing human or other primate DNA sequences (Stanyon et al. 2008; Eder et al. 2003; Tsend-Ayush et al. 2004; Muller et al. 2004). This highlighted the presence of evolutionary chromosomal rearrangements, such as inversions, translocations, fissions/fusions, leading to a repositioning of genes along the chromosomes, and permitting the formation of new syntenic groups.

A number of studies with interphase FISH have shown that chromosome territories are organised in the nucleus on the basis of gene density and GC level (Croft et al. 1999). More precisely, they present a zigzag organisation to position the gene-richest and the gene-poorest regions (corresponding to the GCrichest and the GC-poorest isochores, respectively) in the more internal and peripheral part of the nucleus, respectively (Saccone et al. 2002; Gilbert et al. 2004; Zink 2006; Hepperger et al. 2007; Federico et al. 2008). This also allows for specific compositional properties of the different functional classes of genes (information storage and processing, cellular processes and signalling, and metabolism) observed as a footprint in all mammalian genomes (Bernà et al. 2012). Indeed, it is generally assumed that this particular chromatin organisation corresponds to different transcriptional properties, with the transcriptionally active genes located in the inner part of the nucleus (Croft et al. 1999; Kupper et al. 2007; Bickmore and van Steensel 2013; Cremer et al. 2015). Similarly, some subnuclear compartments are generally associated with gene repression, such as the heterochromatic regions located at the nuclear periphery, or around the nucleoli (Sadoni et al. 1999; Foster and Bridger 2005; Finlan et al. 2008).

Chromosomal rearrangements could determine an alteration in the chromatin organisation in the cell nuclei, possibly affecting proper gene functioning. Indeed, gene expression may be influenced by the position of loci and chromosome bands in the interphase nucleus, as a result of a change in the chromatin architecture (Bridger et al. 2000; Bickmore and van Steensel 2013; Cremer et al. 2015). Gene repositioning in the cell nuclei appears to be correlated, on the one hand to a normal reorganisation of chromatin during cell differentiation (Volpi et al. 2000; Kosak et al. 2002; Szczerbal et al. 2009; Leotta et al. 2014), and on the other hand to the ectopic expression of genes favouring the onset of genetic diseases, as described in patients with childhood leukaemia carrying a translocation between human chromosomes 7 and 12 (Ballabio et al. 2009; Tosi et al. 2015).

In addition to FISH, recently developed molecular procedures such as variations of the chromosome conformation capture method: 3C (chromosome conformation capture), 4C (chromosome conformation 
capture-on-chip), 5C (chromosome conformation capture carbon copy), and Hi-C (high-throughput chromosome conformation capture), have also been used to look at long-range chromatin interaction and three dimensional organisation of the genome (Dekker et al. 2002; Simonis et al. 2006; Dostie et al. 2006; Lieberman-Aiden et al. 2009), providing higher-resolution than FISH (Rao et al. 2014). The Hi-C method demonstrated the presence in the nucleus of two genomic compartments, called A and B (Lieberman-Aiden et al., 2009), organised into Topologically Associated Domains (TADs) of various sizes, located more internally and more peripherally, respectively, in the nucleus (Wang et al. 2016). These highlight the correspondence with the GC-richest and GC-poorest chromosomal band DNA, respectively (Saccone et al. 2002; Jabbari and Bernardi 2017; Stevens et al. 2017). Thus, a high degree of convergence in the organisation of chromosomal DNA in the interphase nucleus, using different methodologies (Hi-C or $3 \mathrm{C} / 4 \mathrm{C} / 5 \mathrm{C}$ on the one hand, and interphase $\mathrm{FISH}$ on the other), was obtained. The two methods, endowed different levels of resolution and different obtainable data-sets, should be considered complementary. Indeed, some results on chromatin topography obtained with 5C or FISH are not compatible, possibly due to the fact that products captured by $3 \mathrm{C}$ are not always closely positioned (Williamson et al. 2014; Fraser et al. 2015).

Currently, 3C-derived technologies were not again applied in non-human primate species, and data on the chromatin organisation in cell nuclei of these species were largely obtained using FISH with a variety of probes on 3D-preserved nuclei. Studies on species belonging to Anthropoidea showed that the radial nuclear location (RNL) of the chromosomes homologous to Hsa (Homo sapiens) 18 and Hsa 19 is largely conserved in the species considered (Tanabe et al. 2002). Moreover, a large degree of RNL conservation has been shown for the chromosomes homologous to Hsa6, Hsa12 and Hsa17 in two New World monkey species, other than H. sapiens (Mora et al. 2006). Furthermore, investigations analysing entire chromosomes, as well as smaller regions, highlighted a conserved radial organisation of chromosomes in the nuclei of primate lymphoblastoid cell lines. This was in agreement with the GClevel/gene density, despite the presence of a variety of evolutionary chromosomal rearrangements, which reshuffled the homologous chromosomes (Neusser et al. 2007). Similar work performed with a set of 60 clones containing very-early and very-late replicating DNA, in cells of human and non-human primate species, demonstrated that gene density and GC-level, but not replication timing, are the key players influencing radial nuclear positioning of a locus (Grasser et al. 2008).

During karyotype reshuffling along different primate lineages, a central role has been represented by centromere repositioning where centromere inactivation and concomitant formation of new centromeres in different sites occurs (Montefalcone et al. 1999; Amor and Choo 2002; Ventura et al. 2004; and 2007; Marshall et al. 2008; Purgato et al. 2015; Tolomeo et al. 2017). One well-documented example is represented by the evolution of the $\mathrm{X}$ chromosome in lemurs. Although the synteny along this chromosome is normally very conserved, its structure was found to be different in two lemur species 
due to new centromere formation in different bands along the chromosome (Ventura et al. 2001). Similar observations have been made in other chromosomes (Stanyon et al. 2008).

In the present study, we extend previous investigations on the interphase chromosomal organisation in primate cell nuclei by means of FISH with BAC probes containing human DNA sequences. We analysed the nuclear location of eighteen loci in eight primate species covering the entire evolutionary tree, namely three Hominoidea species, Homo sapiens, Gorilla gorilla, and Pan troglodytes, two Cercopithecoidea, Macaca mulatta and Cercopithecus aethiops, two Platyrrhini, Callicebus moloch and Callithrix jacchus, and one Prosimii, Lemur catta. The eighteen chromosomal loci belong to five human chromosomes (Hsa2, Hsa3, Hsa6, Hsa 7, Hsa 12) that correspond to homologous chromosomes, in other primate species, with and without repositioning along the chromosome, by evolutionary reshuffling, of the loci considered (depending on the species investigated). The main purpose of our work was to increase knowledge on the impact of chromosomal rearrangements (translocations, inversions, new centromere formations) on the 3D genome organisation, via the analysis of the spatial positioning of relevant genes and chromosomal bands occurring during karyotype evolution. Our data indicate that compositional properties and nuclear location of the chromosomal bands involved in the rearrangements play an important role in the resulting phenotype and in the consequent karyotype evolution. 


\section{Materials and methods}

\section{Genomic features of the human chromosomal bands}

Information on the size, GC-level and replication timing of the human chromosomal bands investigated in this study were obtained from previous reports (Costantini et al. 2007, Woodfine et al. 2004). The relative gene content was accessed through the UCSC Human Genome Browser (HGB) Gateway (http://genome.ucsc.edu/).

\section{Preparation of chromosomes and nuclei}

Human ( $H s a)$ metaphase chromosomes and nuclei were prepared from phytohaemagglutinin (PHA)stimulated peripheral blood lymphocytes of healthy donors. For the gorilla (Gorilla gorilla, Ggo), chimpanzee (Pan troglodytes, Ptr), macaque (Macaca mulatta, Mmu), and common marmoset (Callithrix jacchus, Cja) we used lymphoblastoid cell lines, and for red-bellied titi (Callicebus moloch, Cmo), blue monkey (Cercopithecus aethiops, Cae), and ring-tailed lemur (Lemur catta, Lca) we used fibroblastoid cell lines (all cell lines were kindly provided by Dr. M. Rocchi, University of Bari, Italy). Chromosomes and nuclei were prepared according to standard cytogenetic procedures described previously (Federico et al. 2008). In addition, freshly prepared $4 \%$ buffered paraformaldehyde was used to fix the cells and to allow better spatial preservation of the nuclei as described by others (Solovei et al. 2002; Hepperger et al. 2007).

\section{DNA probes, in situ hybridisation, and detection}

Probes used for FISH were BAC clones containing human DNA fragments (Tab. 1). All probes were kindly provided by Dr. M. Rocchi, University of Bari, Italy. DNA probes were extracted using a commercial kit (Qiagen, Milan, Italy), and were biotin- or digoxigenin-labelled by nick translation (Roche, Mannheim, Germany).

Hybridisation conditions for human chromosomes and nuclei were the same as previously described (Federico et al. 2008). In the case of heterologous FISH on primate chromosomes, with respect to the human, the differences concerned the amount of probe (50-250 ng), incubation time to hybridise, and post-hybridisation washings. Briefly, hybridisation was performed (after a 30 min preannealing step with an excess of unlabelled human Cot- 1 DNA) at $37^{\circ} \mathrm{C}$, in a moist chamber, for $16-36$ hours. Posthybridisation washes were carried out (i) for homologous in situ hybridisations (human probes on human chromosomes and nuclei) at $60^{\circ} \mathrm{C}$ with $0.1 \mathrm{xSSC}(1 \mathrm{xSSC}$ is $0.15 \mathrm{M} \mathrm{NaCl}, 0.015 \mathrm{M}$ sodium citrate), and (ii) for the heterologous hybridisations (human probes on non-human chromosomes and nuclei) at $37-45^{\circ} \mathrm{C}$ with $0.5 \mathrm{xSSC}$. Hybridisation detection of biotin- and digoxigenin-labelled probes was performed using TexasRed-conjugated avidin and Fluorescein-conjugated antibody, respectively. Finally, chromosomes were stained with DAPI. Images were captured using epifluorescence 
microscopy (Olympus AX70) and a Photometrics cooled CCD camera with MacProbe v4.2 software (Applied Imaging, Newcastle, U.K.).

All human DNA probes were tested first on metaphase chromosomes of all the species investigated to verify (i) the location on the homologous chromosomal region in the other primates, as expected on the basis of published data concerning syntenic regions among chromosomes, and (ii) the absence of additional signals on other sites, essential for a reliable analysis in the interphase nuclei.

\section{Localisation of FISH signals in the cell nuclei, and statistical analysis}

The radial nuclear location (RNL) of the hybridised probes was obtained using 2-dimensional (2-D) analysis, as previously described (Federico et al. 2008). Briefly, a high number of hybridised nuclei was randomly captured using MacProbe v4.2 software, and hybridisation signals were then localised in each cell nucleus using a specific computer program (developed in our lab, and freely available upon request). Each hybridisation signal was located in the nucleus using a value corresponding to the ratio of the nuclear radius ( 0 and 1 indicate the centre and the peripheral rim of the nucleus, respectively). The median values +/- Confidence Interval (C.I.) of at least 300 hybridisation signals were then evaluated. Median values lower and higher than 0.65 indicate loci located more internally or peripherally, respectively, in the nuclei. The statistical analyses were carried out using Microsoft Excel and StatView software. 


\section{Results}

\section{Radial nuclear location of BAC probes on human cell nuclei}

For FISH experiments, we selected eighteen loci, from euchromatic regions of the human chromosomes, endowed by different GC-levels, gene-densities, and replication timing. The genomic properties were evaluated in the $2 \mathrm{Mb}$ DNA segment around each probe, and in the chromosomal band containing the probe (Tab. 1). The loci analysed span a wide range of GC-level values, and are located on compositionally different human chromosomal bands (Fig. 1). Eight loci are located in GC-poor bands $(<38 \%$ GC), six in GC-rich bands $(>45 \% \mathrm{GC})$, and the other four in compositionally intermediate bands.

RNL was obtained by in situ hybridisation of the BAC probes on interphase cell nuclei, and by statistical evaluation of data from hundreds of hybridised nuclei. The obtained median values (Fig. 2) showed a range from 0.552 (corresponding to the very GC-rich probe RP11-213E22, located in the $7 \mathrm{q} 22.1$ band) to 0.819 (corresponding to the very GC-poor probe RP11-886P7, located in the 2q22.1

band). Results obtained with the four probes located in chromosome 7 largely corresponded to those previously described (Federico et al. 2008). Generally, BAC probes located in GC-poor or GC-rich bands are located more peripherally or more internally, respectively, in the cell nuclei. Comparisons among peripheral $(\mathrm{RNL}>0.65)$ and inner $(\mathrm{RNL}<0.65)$ loci were always highly statistically significant, except for the RNLs of RP11-102I23 and RP11-148K1 with respect to RP11-825M8, namely between loci very close to the 0.65 value.

The RNLs were correlated to the GC-level, gene-density, and replication timing indicating, as expected, a high level of correlation (Fig. 3). More specifically, the highest correlation level between RNL and GC-level/gene-density/replication timing were obtained with the entire chromosomal band containing the probe (i.e., a chromosomal DNA segment around the probe characterised by a relatively homogeneous composition), regardless of the band's size.

\section{Chromosomal and nuclear location of BAC probes on primates}

We evaluated the RNL, in non-human primate species, of the eighteen probes described above (see Tab. 1). Some of these were located in a chromosomal region involved in evolutionary rearrangements. In some species, the heterologous hybridisation efficiency did not allow for the evaluation of the RNL. In some cases, the background precluded the clear identification of the specific signals, and in other cases the signals were not observed at all or the number of nuclei was not statistically adequate. Figure 4 shows the hybridisation signals in the mitotic chromosomes of the loci in which RNL evaluation was carried out. In general, the human probes were more difficult to analyse when they were located in GC rich regions and hybridised in phylogenetically more distant species that gave multiple signals in 
different chromosomes or no signals at all. Lemur catta had the lowest number of loci with an evaluated RNL.

\section{Human chromosome 2 (Hsa2)}

In human chromosomes, BAC probes RP11-102I23 and RP11-886P7 are located in the 2p11.2 and 2q22 band, respectively. Chromosome $H_{s} a 2$, in the other primates, corresponds to two chromosomes, one homologous to the $p$ arm (and a small part of the $q$ arm) and the other to the remaining $q$ arm (Fig. 4A). This involved a reorganisation of the centromeres, with the emergence of evolutionary new centromeres (ENCs) in Mmu12 and Mmu13. The position of these ENCs corresponds to the loci identified by the BAC probes used here, with the splitting of the hybridisation signal visible in the Mmu12 and Mmu13 chromosomes, and single spots in the homologous chromosomes of the other analysed species (Hsa, Ggo, Cja). RNLs of the RP11-102I23 and RP11-886P7 probes in the four analysed species indicated a peripheral location, with the more internal value observed for the RP11-102I23 locus in Hsa2 (Fig. 5).

\section{Human chromosome 3 (Hsa3)}

In chromosome Hsa3 we analysed five loci: two of them (detected by RP11-655A17 and RP11-227H4 probes) are located close to the centromere in Hsa3, Ptr2, and Ggo2. In Mmu2, Cae22, and Lcal they are in the same relative position but the centromere between them is not present. In Cmo and Cja they are in different chromosomes with the RP11-655A17 conserving the position in a band close to the centromere, and the RP11-227H4 relocated, in one case, to a telomeric position, very distant from the centromere (Cja15). RNLs of these two loci showed a peripheral location in all of the analysed cases (Fig. 5). A similar situation was observed for the BACs RP11-505J9 and RP11-526M23, which showed a peripheral location in all of the analysed cases (Fig. 5). These two loci were always in the same relative position, not closely associated with the centromere, except in Mmu2 and Cae15 where a centromere was present between them (Fig. 4C). The fifth locus (detected by the RP11-313F11 probe) conserved a telomeric position in the analysed species except in Lcal (but in this latter case the number of informative nuclei were low and RNL not determined) (Fig. 4C), and the RNLs always corresponded to the internal part of the nucleus (Fig. 5).

\section{Human chromosome 6 (Hsa6)}

The selected loci from chromosome Hsa6 maintained the same location along the homologous chromosomes Hsa6, Ptr5, Ggo5, Mmu4, and Cja4, even if centromere repositioning occurred in the lineages resulting in Cja and $M m u$. Indeed, the locus identified by the RP11-474A9 probe corresponds to the centromeric region in $M m u 4$, as visible by the split signals in this chromosome (Fig. 4B). RNLs evaluated for these four probes showed a peripheral position for the RP11-55K11, RP11-959I6 and RP11-474A9 probes, and an internal location for the RP11-79O21 probe (Fig. 5). It should be noted, in 
this case, that the RNL of each probe in the different species showed very comparable values, especially with the RP11-959I6 probe with RNLs very close to the median value 0.750 for all the species analysed.

\section{Human chromosome 7 ( $\mathrm{Hsa} 7)$}

Loci from chromosome $\mathrm{Hsa} 7$ present a number of positional features (Fig. 4D). RP11-6A1 is telomeric in Hsa7, Ggo6, and Cae28 and pericentromeric in Cja2. RP11-79O21 is positioned along the chromosomes Hsa7, Ggo6, Mmu2, Cae21, and Cja8, always far from the centromere. RP11-213E22 is positioned in a chromosomal band close to the centromere in Ggo14, Mmu2, and Cae28. 148K1 is always telomeric except in $C j a 8$, where it is near the centromere (Fig. 4D). RNLs of these four loci in all the species analysed are related to the RNLs evaluated in the human cell nuclei and to the GC-level of the human chromosomal band where each locus is located. Namely, RP11-6A1 and RP11-213E22 loci are in the inner part of the nuclei, whereas RP11-79O21 and 148K1 are located peripherally (Fig. 5).

\section{Human chromosome 12 (Hsa12)}

In chromosome Hsa12, we used three loci from the short arm (Fig. 4E). Two of them, RP11-79K20 and RP11-712A21, show the same relative position along the homologous chromosomes Hsa12, Ptr10, Ggo10, Mmu12, Cja9 and Cmo10, but in this latter case a centromere emerged between them. RP11485K 18 is close to the centromere in Hsa12, Ptr10, Ggo10, and Mmu12, and more distant in Cja9 and Cmo10. RNLs were found to be peripheral for RP11-712A21 and RP11-485K18 loci, and in the inner part of the nuclei for RP11-79K20 (Fig. 5), highly correlated to the RNLs evaluated in the human cell nuclei.

On the basis of the RNLs, we can classify the 18 loci into two main categories, one including those located at the nuclear periphery and the other located in the inner part of the nucleus. The first group contains the larger number of loci; the second group contains five loci identified by the probes RP11313F11, RP11-825M8, RP11-6A1, RP11-213E22, and 79K20. Among the loci studied, the latter are endowed, in the human genome, with the highest GC level and gene-density and early replicating during the S-phase of the cell cycle (see Table 1). The RNLs of a probe in the different species were generally very similar, with differences not statistically significant, as evaluated by a two-tailed t-test. Some exceptions concerned probes RP11-102I23, RP11-886P7, RP11-655A7, RP11-227H4, and RP11526M23, which showed a more spread out range in the RNL values, even though the location in the various species was always in the peripheral part of the nucleus. 


\section{Human chromosomal bands and new centromeres}

We analysed the GC-level of the chromosomal bands that are involved in different types of centromerisation events. More precisely, we considered three different types of human chromosomal bands, in reference to the different centromere features: (i) bands that in the ancestral chromosomes were occupied by a centromere, the ancestral centromere (AC), subsequently lost and now absent in the present chromosome (Stanyon et al. 2008), (ii) bands that in other homologous primate chromosomes correspond to an evolutionary new-centromere (ENC), which appeared concomitantly to the AC inactivation or to a centromere occurrence in an acentric chromosome fragment (Stanyon et al. 2008), and (iii) bands frequently related to the formation of human constitutional new-centromeres (HCN), as a consequence of chromosomal rearrangements (Marshall et al. 2008).

The GC-level of ENC, HCN and AC were evaluated on 21, 201, and 7 human chromosomal bands (see Fig. 1) and showed a mean value of $37.6 \%, 41.1 \%$ and $40.3 \%$ respectively (Fig. 6). In the case of HCN the above 201 bands correspond to all bands (at a resolution of 850 bands per haploid genome) included in the $30 \mathrm{HCN}$ regions (Marshall et al. 2008 and Fig. 1). The average GC-level of the chromosomal bands with ENC is statistically different with respect to the GC-level of $\mathrm{HCN}$ ( $\mathrm{P}<0.0001$, as evaluated by a two-tailed t-test). The AC-related bands did not show differences with HCN or with ENC, but in this case it should be stressed that the number of AC bands was too low (seven in total) for a reliable statistical evaluation. In addition to the GC level of the bands related to $\mathrm{ENC}, \mathrm{HCN}$ and $\mathrm{AC}$, we analysed the $\mathrm{GC} \%$ of the bands belonging to the human chromosome 19 and 22, because in these chromosomes no HCNs or ENCs were described. The average GC-level of the chromosomal bands of Hsa19 is $47.8 \%$ and of Hsa22 is $47.2 \%$. These values are not statistically different from each other, but are very statistically different with respect to the three types of bands considered here (ENC, HCN, AC) (see table in Fig. 6). 


\section{Discussion}

In the present work, we evaluated the radial nuclear location of eighteen chromosomal regions in humans and in seven other primate species. A number of these regions show a reshuffling in the homologous chromosomes of the present primate karyotypes. Additionally, we analysed the genomic properties related to the GC-level. We also focused our attention on the chromosomal bands frequently involved in new-centromere formation, either in the case of pathological human conditions (human constitutional centromeres, $\mathrm{HCN}$ ) or during chromosomal evolution (evolutionary new centromeres, ENC, and ancestral centromeres, AC). The aim was to understand the mechanisms underlying chromosomal rearrangements leading to changes associated with their evolutionary success, and whether these mechanisms could be considered different with respect to those related to chromosomal abnormalities, described in the scientific literature and involved in human pathological conditions.

The results we present here show that the nuclear location of the eighteen loci analysed is generally unchanged in the cells of the primate species, regardless of whether or not the corresponding chromosomal band was involved in evolutionary chromosomal reshuffling. This indicates that evolutionary rearrangements of the chromosomes do not affect the functional properties of the involved regions.

\section{Evolutionary chromosomal rearrangements}

The organisation of chromosomes and genes within the nucleus is associated with different structural and functional properties of the genome, such as GC level, replication timing, and transcriptional activity. These characteristics define two main types of genomic regions that are distributed unequally, not only along the metaphase chromosomes (see Fig. 1) but also in the interphase nuclei as first demonstrated by molecular cytogenetic methods (Croft et al. 1999; Saccone et al. 2002; Gilbert et al. 2004) and later on confirmed by Hi-C (Lieberman-Aiden et al. 2009). Thus, two nuclear compartments with opposite features were demonstrated: one corresponding to the GC-richest isochore families (according to the nucleotide composition) or to the A compartment (according to the Hi-C method) is localised in the nuclear interior and have the highest gene density, a more open chromatin structure, replication at the onset of the $\mathrm{S}$ phase, and higher transcriptional activity; the other corresponds to the GC-poorest isochore families (according to the nucleotide composition) or to the $\mathrm{B}$ compartment (according to the Hi-C method) and is endowed with opposite features. This latter compartment, further to a preferential location at the nuclear periphery and around the nucleoli, is enriched in lamina associated domains (LADs) (Kind et al. 2013; Cremer et al. 2015; Bernardi 2015; Stevens et al. 2017).

Chromosomal rearrangements may happen between bands with the same or different compositional features, determining different outcomes. When a chromosomal rearrangement involves chromosomal bands with the same compositional features and endowed by a similar RNL, the genes located in the rearranged regions should maintain, presumably, their nuclear position and their transcriptional 
properties. This type of rearrangement, which is potentially neutral to the phenotype, could be preserved in the population and thus become important in chromosomal evolutionary modifications. In this regard, orthologous genes in different species should be located in the same nuclear compartment, in order to preserve the optimal conditions for gene regulation and transcription.

In the present study, we analysed, in addition to some genomic properties, the nuclear location of eighteen human chromosomal bands and the corresponding homologous regions in other primate species to understand if the above assumption could be verified. We obtained the nuclear location, in the human PHA-stimulated lymphocytes (Fig. 2), of 18 BAC probes located in human chromosomal bands with various compositional properties (Tab. 1 and Fig. 1). The GC-rich and GC-poor loci were generally localised in the more internal and more peripheral parts of the cell nucleus, corresponding to the Hi-C A and B compartments, respectively, with the RNL of the probes on chromosome Hsa7 in accordance with previous data (Federico et al. 2008). Our present results also confirmed the highest level of correlation between RNL with gene density and replication timing of the entire band in which each probe is localised (see Fig. 3).

During the evolutionary history of primate chromosomes, some of the bands we studied here modified their position along the metaphase chromosome, while others maintained the same position, even if some current homologous chromosomes show different sizes (e.g., the band 3q29 identified by BAC RP11-313F11 is generally located in a telomeric position except in $L c a$ ). Our results showed a generally conserved RNL for the probes analysed; namely each probe is located in the same nuclear compartment (inner/peripheral nuclear position or Hi-C A/B compartment) across different species, independent of the evolutionary chromosomal reshuffling occurring in the homologous chromosomes. This indicates that each chromosomal region maintains similar RNL and possibly similar gene activity in different primate lineages, in both Old and New World monkeys. Indeed, as mentioned above, the conserved RNL of a specific chromosomal region should be a good indication of the preservation of the region's function, and this is independent of the position along the metaphase chromosome. Moreover, some chromosomal regions, such as those of chromosome $H s a 6$ and $H s a 7$, showed a very high level of conservation of the nuclear location in the investigated species (see Fig. 5).

The data presented here, obtained on chromosomes showing different types of evolutionary rearrangements, indicate that the impact of inversions or translocations on the gene nuclear location is very similar; namely none of the chromosomal loci analysed here changed their nuclear location in the different species. It should be stressed that the large amount of data was obtained with lymphoblastoid cells, endowed with a different type of DNA distribution in the nucleus with respect to the fibroblast cells (Neusser et al. 2007; Grasser et al. 2008). However, even if little data were obtained here with fibroblastoid cells (6, 5, 2 probes for Cae, Cmo, Lca respectively), the variability in the nuclear location for a defined locus in the different species was very low. The higher variability in the RNL was observed for the two regions of chromosome Hsa2 (2p11.2 and 2q22.1 bands) and three regions of 
chromosome Hsa3 (3p12.1, 3q12.1, 3q26 bands), but the nuclear position in the species analysed was always higher than 0.65 (see Fig. 5), namely values indicating the location in the peripheral compartment of the cell nucleus. This is slightly different with respect to previous work that used fibroblast cell nuclei and showed that inversions seem to influence nuclear topology to a greater extent than translocations, even if this was generally seen with some GC-poor/late replicating regions that were observed more internally in the human fibroblast nuclei, but with a more peripheral location in the orangutan fibroblasts (Grasser et al. 2008).

\section{Pathological chromosomal rearrangements}

Evolutionary chromosomal rearrangements not affecting the RNL of genes should be clearly distinguished from chromosomal abnormalities observed in nuclei of diseased human cells. Correct reorganisation of chromatin during cell differentiation leads to a functional gene repositioning according to the cell type considered (Kosak et al. 2002; Szczerbal et al. 2009; Leotta et al. 2014). This process may be altered if chromosomal rearrangements determine the alteration of the nuclear position of specific genes, leading to genetic diseases (Bickmore and van der Maarel 2003; Foster and Bridger 2005; Ono et al. 2007; Ballabio et al. 2009; Tosi et al. 2015).

We suggest that evolutionary chromosomal rearrangements differ from those found in human genetic diseases because the latter determines a gene structure disruption or a modification of the nuclear compartment where a gene is located (peripheral/inner, or Hi-C B/A), namely the repositioning from the peripheral to the inner part of the nucleus (or Hi-C B vs A compartment) or vice versa. In fact, the results reported here indicate that evolutionary chromosomal rearrangements typically do not affect the nuclear location of the involved regions and that we can consider them neutral mutations that escape natural selection. This is clearly in contrast, from an evolutionary point of view, to the clinical chromosomal rearrangements that lead to a radial nuclear repositioning of the loci in different compartments and on which natural selection acts negatively, for example by elimination of the cell from the organism, eliminating the organism itself from the population, or by reducing fitness. This could be due to a rearrangement between two chromosomal bands with very different genomic properties, and with a nuclear position in different functional compartments, which determines the joining of two regions allowing a nuclear repositioning of the involved bands (Tosi et al. 2015). This is in line with the genomic instability described in those rearranged chromosomal regions endowed with a switch in replication timing and GC\% (Watanabe and Maekawa 2013).

\section{New centromere formation}

Similar reasoning can be used to explain the phenomenon of neocentromerisation. To date, no sequence specifically involved in the formation of new centromeres has been identified and the origin of a neocentromere appears to be associated with epigenetic phenomena that are not yet well understood. An 
evolutionary neocentromere recently studied in the macaque showed the absence of a nucleotide sequence modification with respect to the original sequence, with the only difference being a higher degree of compaction of the neocentromerised region (Tolomeo et al. 2017).

Considering current data on the genomic properties of the $\mathrm{HCN}$, ENC, and AC regions (see Fig. 6), it can be hypothesised that a neocentromere can form in every chromosomal region. However, depending on the genomic features of the involved region, the new centromeres will have a different fate over time: a new centromere emerging in a very GC-poor region, depleted in genes, normally located in the most peripheral part of the cell nucleus and in a very compact form, has a high likelihood of staying in the population as an "asymptomatic" polymorphism. In other words, it will not lead to any significant phenotypic effect in the cell or the organism in which it formed, as described for example in the neocentromeres observed in phenotypically normal human individuals (Ventura et al. 2004). If the new centromeres originate in regions with very high GC levels, with very high gene density and a location in the internal, transcriptionally-active nuclear compartment, the chance of being retained is very low, because of its possible negative effect on fitness. In support of this hypothesis, there is no report, to our knowledge, of neocentromerisation events in regions with the above features, such as in chromosomes Hsa 19 and Hsa22 (Marshall et al. 2008; Stanyon et al. 2008). Thus, without any way to verify this, we can only speculate that neocentromere formation in these chromosomes would have a very short life, and would be lethal for the cell in which they appear. The formation of neocentromeres in chromosomal regions with intermediate compositional features, compared to those described above, may be associated with a variety of damaging consequences. Thus, these will be observed in subjects with various types of pathologies associated with the presence of the so-called human constitutional neocentromeres (Amor and Choo 2002; Marshall et al. 2008).

\section{Conclusion}

Metaphase chromosomes can modify their size and structure by a variety of modes, such as translocations, fissions, fusions and inversions, determining a wide range of phenotypic consequences, from no evident effect to very relevant clinical symptoms. Considering previous literature and our present data, this could be related to the compositional features of the chromosomal regions involved in the rearrangements. When these rearrangements do not affect gene structure and leave unaltered the preexisting nuclear location, we can assume that they do not lead to alterations of gene expression patterns and have a high probability of being evolutionarily conserved. Indeed, such "asymptomatic" or "neutral" rearrangements could be preserved by a neutral mode of selection. In this case, chromosomes can modify their size and form, and chromosomal bands can be repositioned along a chromosome if their organisation in the cell nucleus remains unchanged. This type of event is obviously very different from those resulting in genetic diseases, that instead lead to alterations in the higher order organisation of the chromatin and changes in nuclear gene positioning. 
Along this line of evidence, new centromere formation and, more specifically, its evolutionary success, depends on the type of chromosomal region in which it occurs. If the region has the same genomic properties of the canonical centromeric region (low GC-level, absence or few genes, late replication during the S-phase of the cell cycle, nuclear location in the peripheral compartment), the new centromere will have a high probability of being evolutionarily conserved. On the contrary, the new centromeres formed in those regions where the functional impact is very detrimental to cell viability are rapidly eliminated from the population, as in the case of rearrangements between chromosomal bands with very different genomic properties.

\section{Acknowledgements}

The authors wish to thank the anonymous reviewers for constructive comments. This work was supported by P.R.A. to SS and F.I.R. to VF from the University of Catania. 


\section{References}

Amor DJ, Choo KH (2002) Neocentromeres: role in human disease, evolution, and centromere study. Am J Hum Genet 71:695-714

Ballabio E, Cantarella CD, Federico C, Di Mare P, Hall G, et al. (2009) Ectopic expression of the HLXB9 gene is associated with an altered nuclear position in $t(7 ; 12)$ leukaemias. Leukemia 23:1179-1182

Bernà L, Chaurasia A, Angelini C, Federico C, Saccone S, et al. (2012) The footprint of metabolism in the organization of mammalian genomes. BMC GENOMICS, vol. 13, p. 174-186

Bernardi G (2015) Chromosome architecture and genome organization. PLoS ONE 10(11)e0143739. doi:10.1371/journal.pone.0143739

Bickmore WA, van der Maarel SM (2003) Perturbations of chromatin structure in human genetic disease: recent advances. Human Molecular Genetics 12:207-213

Bickmore, WA, van Steensel B. (2013) Genome architecture: domain organization of interphase chromosomes. Cell 152:1270-1284.

Bridger JM, Boyle S, Kill IR, Bickmore WA (2000) Re-modelling of nuclear architecture in quiescent and senescent human fibroblasts. Curr Biol 10:149-152

Costantini M, Clay O, Federico C, Saccone S, Auletta F, et al. (2007) Human chromosomal bands: nested structure, high definition map and molecular basis. Chromosoma 116:36-49

Cremer T, Cremer M, Hübner B, Strickfaden H, Smeets D, et al. (2015) The 4D nucleome: Evidence for a dynamic nuclear landscape based on co-aligned active and inactive nuclear compartments. FEBS Lett. 589 (20 Pt A), 2931-2943.

Croft JA, Bridger JM, Boyle S, Perry P, Teague P, et al. (1999) Differences in the localization and morphology of chromosomes in the human nucleus. J Cell Biol 145:1119-1131

Dekker J, Rippe K, Dekker M, Kleckner N. (2002) Capturing chromosome conformation. Science 295:1306-1311.

Dostie J, Richmond TA, Arnaout RA, Selzer RR, Lee WL, et al. (2006) Chromosome Conformation Capture Carbon Copy (5C): a massively parallel solution for mapping interactions between genomic elements. Genome Res 6:1299-1309.

Eder V, Ventura M, Ianigro M, Teti M, Rocchi M, et al. (2003) Chromosome 6 Phylogeny in Primates and Centromere Repositioning. Mol Biol Evol 20:1506-1512

Federico C, Cantarella CD, Di Mare P, Tosi S, Saccone S (2008) The radial arrangement of the human chromosome 7 in the lymphocyte cell nucleus is associated with chromosomal band gene density. Chromosoma 117:399-410

Finlan LE, Sproul D, Thomson I, Boyle S, Kerr E, et al. (2008) Recruitment to the nuclear periphery can alter expression of genes in human cells. PLoS Genet 4(3):e1000039. doi: 10.1371/journal.pgen.1000039

Foster HA, Bridger JM (2005) The genome and the nucleus: a marriage made by evolution. Genome organization and nuclear architecture. Chromosoma 114:212-229

Fraser J, Williamson I, Bickmore WA, Dostie J (2015) An Overview of Genome Organization and How We Got There: from FISH to Hi-C. Microbiol Mol Biol Rev 79: 347-372

Gilbert N, Boyle S, Fiegler H, Woodfine K, Carter NP, et al. (2004) Chromatin Architecture of the Human Genome: Gene-Rich Domains Are Enriched in Open Chromatin Fibers. Cell 118:555-566

Grasser F, Neusser M, Fiegler H, Thormeyer T, Cremer M, P. et al. (2008) Replication-timingcorrelated spatial chromatin arrangements in cancer and in primate interphase nuclei. J Cell Science 121:1876-1886

Hepperger C, Otten S, von Hase J, Dietzel S. (2007) Preservation of large-scale chromatin structure in FISH experiments. Chromosoma 116:117-133 
Ijdo JW, Baldini A, Ward DC, Reeders ST, Wells RA (1991) Origin of human chromosome 2: An ancestral telomere-telomere fusion. Genetics 88:9051-9055

Jabbari K, Bernardi G. (2017) An Isochore Framework Underlies Chromatin Architecture. PLoS ONE 12(1): e0168023. doi:10.1371/journal.pone.0168023.

Kind J, Pagie L, Ortabozkoyun H, Boyle S, de Vries SS, et al. (2013) Single-cell dynamics of genomenuclear lamina interactions. Cell 153:178-192.

Kosak ST, Skok JA, Medina KL, Riblet R, Le Beau MM, et al. (2002) Subnuclear compartmentalization of immunoglobulin loci during lymphocyte development. Science 296:158-162

Kupper K, Kolbl A, Biener D, Dittrich S, von Hase J, et al. (2007) Radial chromatin positioning is shaped by local gene density, not by gene expression. Chromosoma 116:285-306

Leotta CG, Federico C, Brundo MV, Tosi S, Saccone S. (2014) HLXB9 gene expression, and nuclear location during in vitro neuronal differentiation in the SK-N-BE neuroblastoma cell line. PLoS ONE 9(8): e105481. doi:10.1371/journal.pone.0105481

Lieberman-Aiden E, van Berkum NL, Williams L, Imakaev M, Ragoczy T, et al. (2009) Comprehensive mapping of long-range interactions reveals folding principles of the human genome. Science 326:289-293.

Marshall OJ, Chueh AC, Wong LH, Choo KH. (2008) Neocentromeres: new insights into centromere structure, disease development, and karyotype evolution. Am J Hum Genet. 82:261-82. doi: 10.1016/j.ajhg.2007.11.009.

Montefalcone G, Tempesta S, Rocchi M, Archidiacono N (1999) Centromere repositioning. Genome Res 9:1184-1188

Mora L, Sánchez I, Garcia M, Ponsà M (2006) Chromosome territory positioning of conserved homologous chromosomes in different primate species. Chromosoma 115: 367-375.

Muller S, Finelli P, Neusser M, Wienberg J (2004) The evolutionary history of human chromosome 7. Genomics 84:458-467

Müller S, Stanyon R, O'Brien PCM, Ferguson-Smith MA, Plesker R, et al. (1999) Defining the ancestral karyotype of all primates by multidirectional chromosome painting between tree shrews, lemurs and humans. Chromosoma 108:393-400

Neusser M, Schubel V, Koch A, Cremer T, Müller S (2007) Evolutionary conserved, cell type and species-specific higher order chromatin arrangements in interphase nuclei of primates. Chromosoma 116:307-320

Ono A, Kono K, Ikebe D, Muto A, Sun J, et al. (2007) Nuclear Positioning of the BACH2 Gene in BCR-ABL Positive Leukemic Cells. Genes Chromosomes \& Cancer 46:67-74

Purgato S, Belloni E, Piras FM, Zoli M, Badiale C, et al. (2015) Centromere sliding on a mammalian chromosome. Chromosoma 124:277-287. doi: 10.1007/s00412-014-0493-6.

Rao SS, Huntley MH, Durand NC, Stamenova EK, Bochkov ID, et al. (2014) A 3D map of the human genome at kilobase resolution reveals principles of chromatin looping. Cell 159:1665-1680.

Saccone S, Federico C, Bernardi G (2002) Localization of the gene-richest and the gene-poorest isochores in the interphase nuclei of mammals and birds. Gene 300:169-178

Sadoni N, Langer S, Fauth C, Bernardi G, Cremer T, et al. (1999) Nuclear organization of mammalian genomes: polar chromosome territories build up functionally distinct higher order compartments. J Cell Biol 146:1211-1226

Seuànez HN (1979) The Phylogeny of Human Chromosomes. Springer, Berlin.

Simonis M, Klous P, Splinter E, Moshkin Y, Willemsen R, et al. (2006) Nuclear organization of active and inactive chromatin domains uncovered by chromosome conformation capture-on-chip (4C). Nat Genet. 38:1348-1354. 
Solovei I, Cavallo A, Schermelleh L, Jaunin F, Scasselati C, et al. (2002) Spatial Preservation of Nuclear Chromatin Architecture during Three- Dimensional Fluorescence in Situ Hybridization (3D-FISH). Exp Cell Res 276:10-23

Stanyon R, Rocchi M, Capozzi O, Roberto R, Misceo D, et al. (2008) Primate chromosome evolution: Ancestral karyotypes, marker order and neocentromeres. Chromosome Res 16:17-39

Stevens TJ, Lando D, Basu S, Atkinson LP, Cao Y, et al. (2017) 3D structures of individual mammalian genomes studied by single-cell Hi-C. Nature 544:59-64. doi: 10.1038/nature21429.

Szczerbal I, Foster HA, M. Bridger JM (2009) The spatial repositioning of adipogenesis genes is correlated with their expression status in a porcine mesenchymal stem cell adipogenesis model system. Chromosoma 118:647-663

Tanabe H, Muller S, Neusser M, von Hase J, Calcagno E, et al. (2002) Evolutionary conservation of chromosome territory arrangements in cell nuclei from higher primates. Proc Natl Acad Sci USA 99:4424-4429

Tolomeo D, Capozzi O, Stanyon RR, Archidiacono N, D'Addabbo P, et al. (2017) Epigenetic origin of evolutionary novel centromeres. Sci Rep. 3:41980. doi: 10.1038/srep41980.

Tosi S, Mostafa Kamel Y, Owoka T, Federico C, Truong TH, et al. (2015) Paediatric acute myeloid leukaemia with the $\mathrm{t}(7 ; 12)(\mathrm{q} 36 ; \mathrm{p} 13)$ rearrangement: a review of the biological and clinical management aspects. Biomarker Research 3:21. DOI: 10.1186/s40364-015-0041-4

Tsend-Ayush E, Grutzner F, Yue Y, Grossmann B, Hansel U, et al. (2004) Plasticity of human chromosome 3 during primate evolution. Genomics 83:193-202

Ventura M, Antonacci F, Cardone M.F, Stanyon R, D’Addabbo P, et al. (2007) Evolutionary Formation of new centromeres in Macaque. Science 316:243-246

Ventura M, Archidiacono N, Rocchi M (2001) Centromere emergence in evolution. Genome Res 11:595-599

Ventura M, Weigl S, Carbone L, Cardone MF, Misceo D, et al. (2004) Recurrent sites for new centromere seeding. Genome Res 14:1696-1703

Volpi EV, Chevret E, Jones T, Vatcheva R, Williamson J, et al. (2000) Large-scale chromatin organization of the major histocompatibility complex and other regions of human chromosome 6 and its response to interferon in interphase nuclei. J Cell Science 113:1565-1576

Wang S, Su JH, Beliveau BJ, Bintu B, Moffitt JR, et al. (2016) Spatial organization of chromatin domains and compartments in single chromosomes. Science 353, 598-602.

Watanabe Y, Maekawa M. (2013) R/G-band boundaries: genomic instability and human disease. Clin Chim Acta 419:108-12. doi: 10.1016/j.cca.2013.02.011.

Williamson I, Berlivet S, Eskeland R, Boyle S, Illingworth RS, et al. (2014) Spatial genome organization: contrasting views from chromosome conformation capture and fluorescence in situ hybridization. Genes \& Development 28:2778-2791

Woodfine K, Fiegler H, Beare DM, Collina JE, McCann OT, et al. (2004) Replication timing of the human genome. Human Molecular Genetics 13:191-202

Zink D (2006) The temporal program of DNA replication: new insights into old questions. Chromosoma 115:273-287 
Tab. 1. Description of the probes.

\begin{tabular}{|c|c|c|c|c|c|c|c|c|c|}
\hline \multirow{2}{*}{ Ref. } & \multirow{2}{*}{ BAC probe } & \multirow{2}{*}{$\begin{array}{c}\text { Probe } \\
\text { location } \\
\text { (band) }\end{array}$} & \multirow{2}{*}{$\begin{array}{c}\text { Probe } \\
\text { position } \\
(\mathrm{Mb})^{(1)}\end{array}$} & \multicolumn{3}{|c|}{$2 M b^{(2)}$} & \multicolumn{3}{|c|}{ Band $^{(3)}$} \\
\hline & & & & GC\% & Genes/Mb & RT & GC\% & Genes/Mb & RT \\
\hline 1 & RP11-102I23 & $2 \mathrm{p} 11.2$ & 86.7 & 433.2 & 13.5 & 1.59 & 433.1 & 12.4 & 1.49 \\
\hline 2 & RP11-886P7 & $2 q 22.1$ & 138.3 & 37.4 & 2.5 & 1.26 & 36.4 & 1.0 & 1.27 \\
\hline 3 & RP11-655A17 & $3 p 12.1$ & 87.2 & 35.5 & 4.0 & 1.22 & 35.4 & 1.0 & 1.19 \\
\hline 4 & RP11-227H4 & $3 q 12.1$ & 99.8 & 35.9 & 4.0 & 1.55 & 38.1 & 7.6 & 1.24 \\
\hline 5 & RP11-505J19 & $3 q 24$ & 150.0 & 37.3 & 10.0 & 1.19 & 36.2 & 2.7 & 1.28 \\
\hline 6 & RP11-526M23 & $3 q 26.1$ & 166.9 & 34.2 & 2.0 & 1.19 & 35.4 & 2.4 & 1.23 \\
\hline 7 & RP11-313 F11 & $3 q 29$ & 197.0 & 45.8 & 17.5 & 1.92 & 45.3 & 17.6 & 1.65 \\
\hline 8 & RP11-55K11 & $6 \mathrm{p} 22.1$ & 26.4 & 40.2 & 31.0 & 1.43 & 40.2 & 20.0 & 1.45 \\
\hline 9 & RP11-825M8 & $6 \mathrm{p} 21.1$ & 44.0 & 47.3 & 25.5 & 1.84 & 46.3 & 25.8 & 1.70 \\
\hline 10 & RP11-959I6 & $6 q 16.1$ & 96.8 & 36.1 & 4.0 & 1.11 & 35.5 & 1.6 & 1.26 \\
\hline 11 & RP11-474A9 & $6 q 24.3$ & 145.7 & 37.2 & 2.5 & 1.47 & 37.7 & 4.1 & 1.39 \\
\hline 12 & RP11-6A1 & $7 \mathrm{p} 22.3$ & 2.1 & 53.7 & 15.5 & 2.00 & 53.4 & 14.3 & 1.92 \\
\hline 13 & RP11-79O21 & $7 \mathrm{p} 21.3$ & 12.1 & 35.9 & 3.0 & 1.19 & 36.2 & 2.8 & 1.30 \\
\hline 14 & RP11-213E22 & $7 q 22.1$ & 100.1 & 49.3 & 35.0 & 2.08 & 48.6 & 23.2 & 1.71 \\
\hline 15 & RP11-148K1 & $7 \mathrm{q} 36.1$ & 150.4 & 46.6 & 22.5 & 1.14 & 45.4 & 15.2 & 1.59 \\
\hline 16 & RP11-79K20 & $12 \mathrm{p} 13.33$ & 1.2 & 43.0 & 10.5 & 1.75 & 45.8 & 15.3 & 1.69 \\
\hline 17 & RP11-712A21 & $12 \mathrm{p} 12.3$ & 18.9 & 37.3 & 3.0 & 1.20 & 36.8 & 6.4 & 1.40 \\
\hline 18 & RP11-485K18 & $12 \mathrm{p} 11.22$ & 28.3 & 37.7 & 5.0 & 1.21 & 38.0 & 8.5 & 1.49 \\
\hline
\end{tabular}

(1) Distance of probe from telomeric end of $\mathrm{p}$ arm. (2) Features of the $2 \mathrm{Mb}$ genomic DNA around the probe. (3) Features of the chromosomal band containing the probe. GC\%: guanine+citosine percentage. RT: replication timing from Woodfine et al. 2004: higher values indicate earlier replication timing. 


\section{Figures}

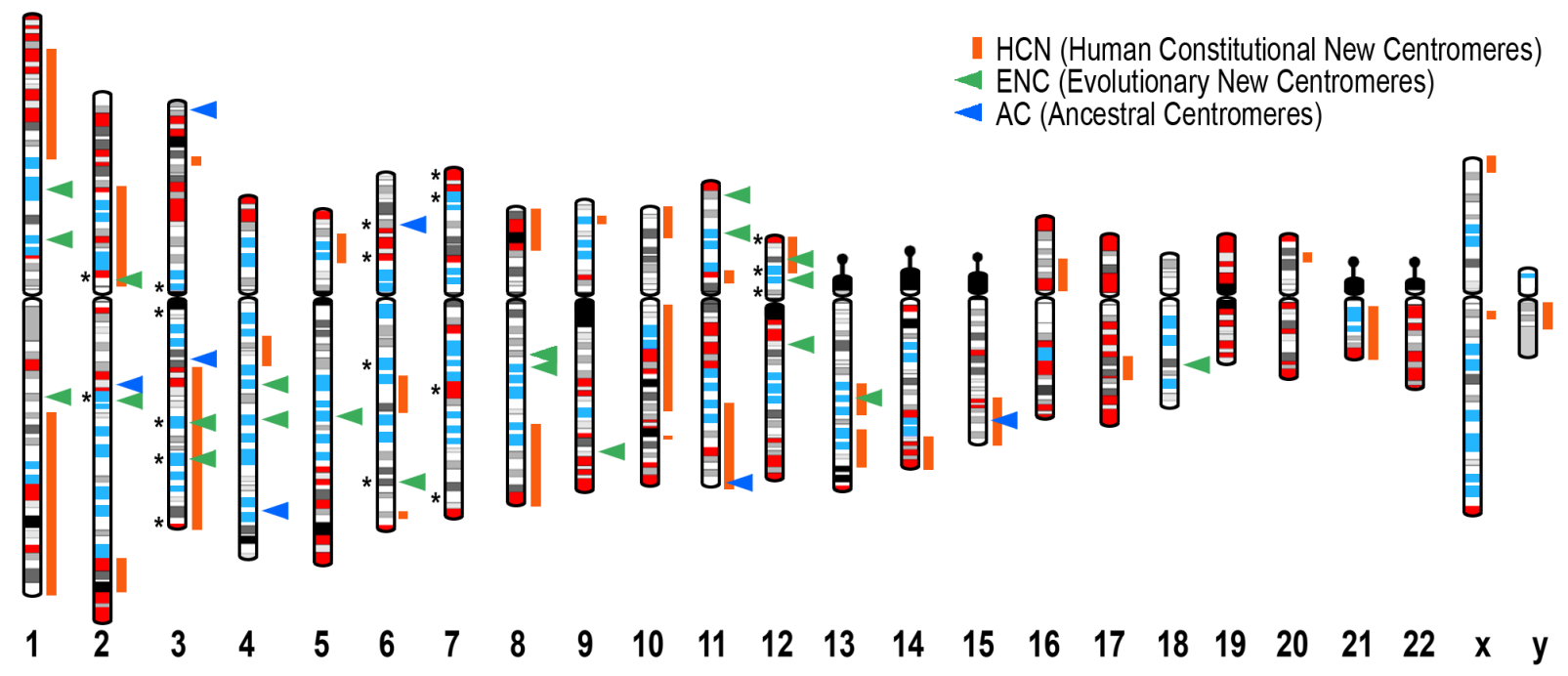

Figure 1. Compositional properties of the human chromosomes. Ideograms of the human chromosomes at a resolution of 850 bands per haploid genome with the GC-richest and GC-poorest bands highlighted (red and blue, respectively) as previously described (Saccone et al. 2002). The asterisks on the left of each chromosome indicate the position of the BAC probes used in the present work (see Tab. 1). HCN, human constitutional new-centromeres (orange vertical bars): chromosomal bands corresponding to sites of recurrent new-centromere formation observed in human cells from subjects with pathological conditions (from Marshall et al. 2008). ENC, evolutionary new-centromeres (green arrows): chromosomal bands corresponding to centromeres in the homologous chromosomes of other primate species (Stanyon et al. 2008). AC, ancestral centromeres, (blue arrows): chromosomal bands corresponding to the centromere site in the ancestral chromosome (Stanyon et al. 2008). 


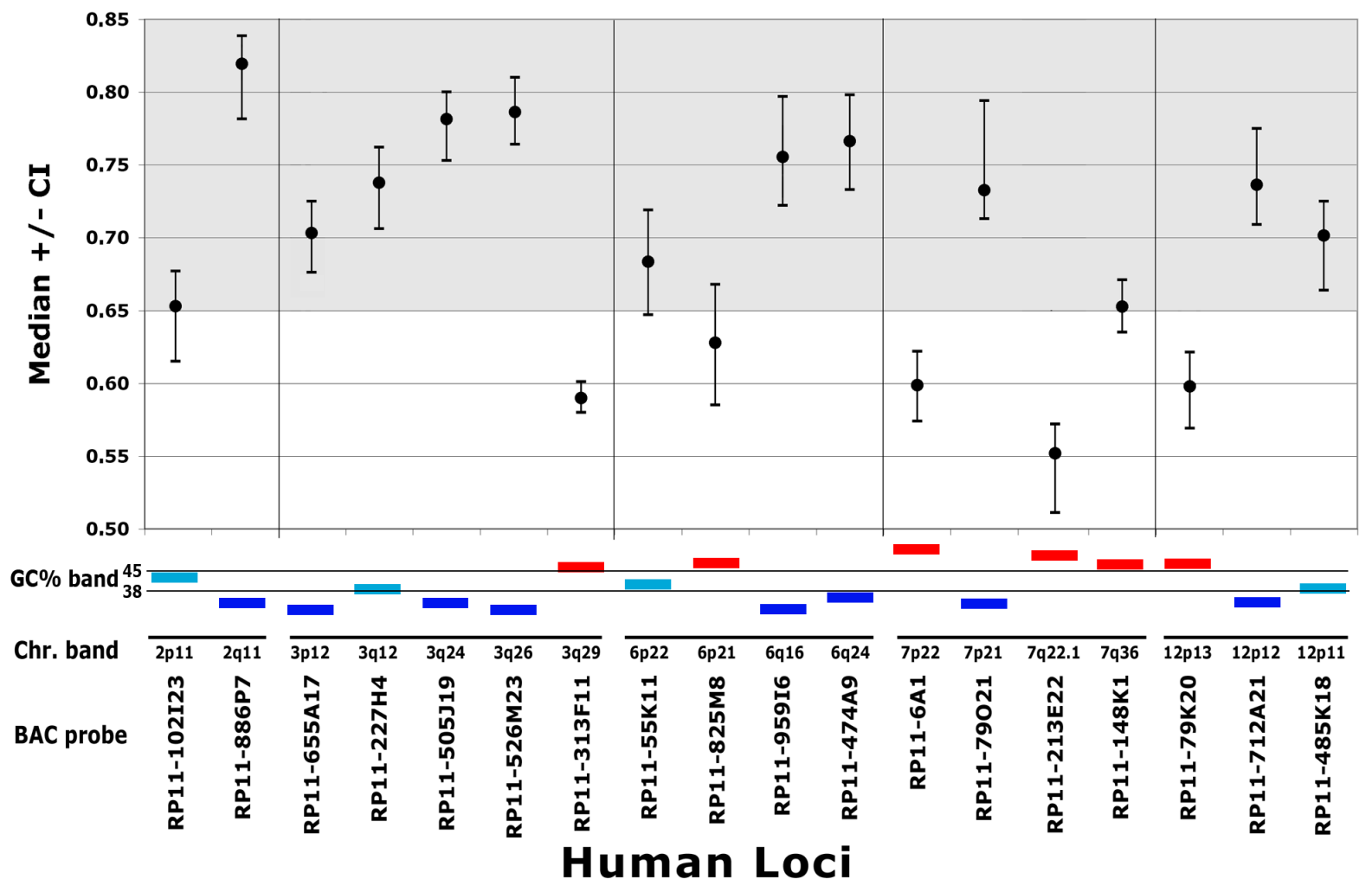

Figure 2. Radial nuclear location of BAC probes in human cell nuclei. The nuclear position of each human BAC probe listed in Table 1 was statistically defined by the median value of more than 300 hybridisation signals localised in the nuclei, as previously described (Federico et al. 2008). On the Yaxis the median values are indicated, along with the relative confidence interval $(\mathrm{CI})$, of each probe. Median values higher and lower than 0.65 indicate probes located at the periphery and in the inner part of the cell nucleus, respectively. Indicated on the $\mathrm{X}$-axis are the BAC probes and the human chromosomal band in which they are located. The GC\% of each band containing the probe is graphically shown: 38 and 45 indicate the GC\% delimitating GC-poor / intermediate / GC-rich bands. GC-rich, intermediate and GC-poor bands are indicated by red, light blue and blue bars, respectively. 


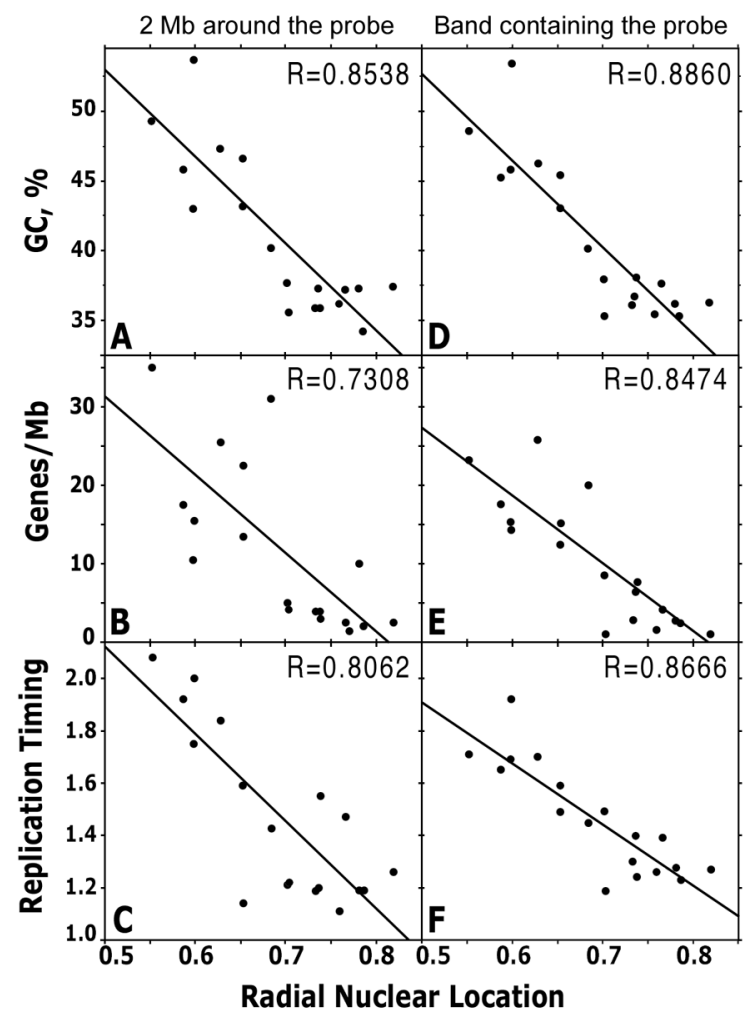

Figure 3. Correlations between RNL of BAC probes on human lymphocytes and GC level, gene density, and replication timing. $\mathrm{A}, \mathrm{B}$, and $\mathrm{C}$ show the correlation between the RNL of each probe and GC level, gene density, and replication timing, respectively, of the $2 \mathrm{Mb}$ DNA segment surrounding the probe. D, E, and F show the correlation between the RNL of each probe and GC level, gene density, and replication timing, respectively, of the chromosomal band containing the probe. $\mathrm{R}$ is the correlation coefficient. The RNL of each locus corresponds to the median value shown in Figure 2. Gene densities and GC level data were obtained from the human genome assembly available in the UCSC Genome Browser (http://genome.ucsc.edu/). The replication timings were those previously described by Woodfine and colleagues (Woodfine et al. 2004). 

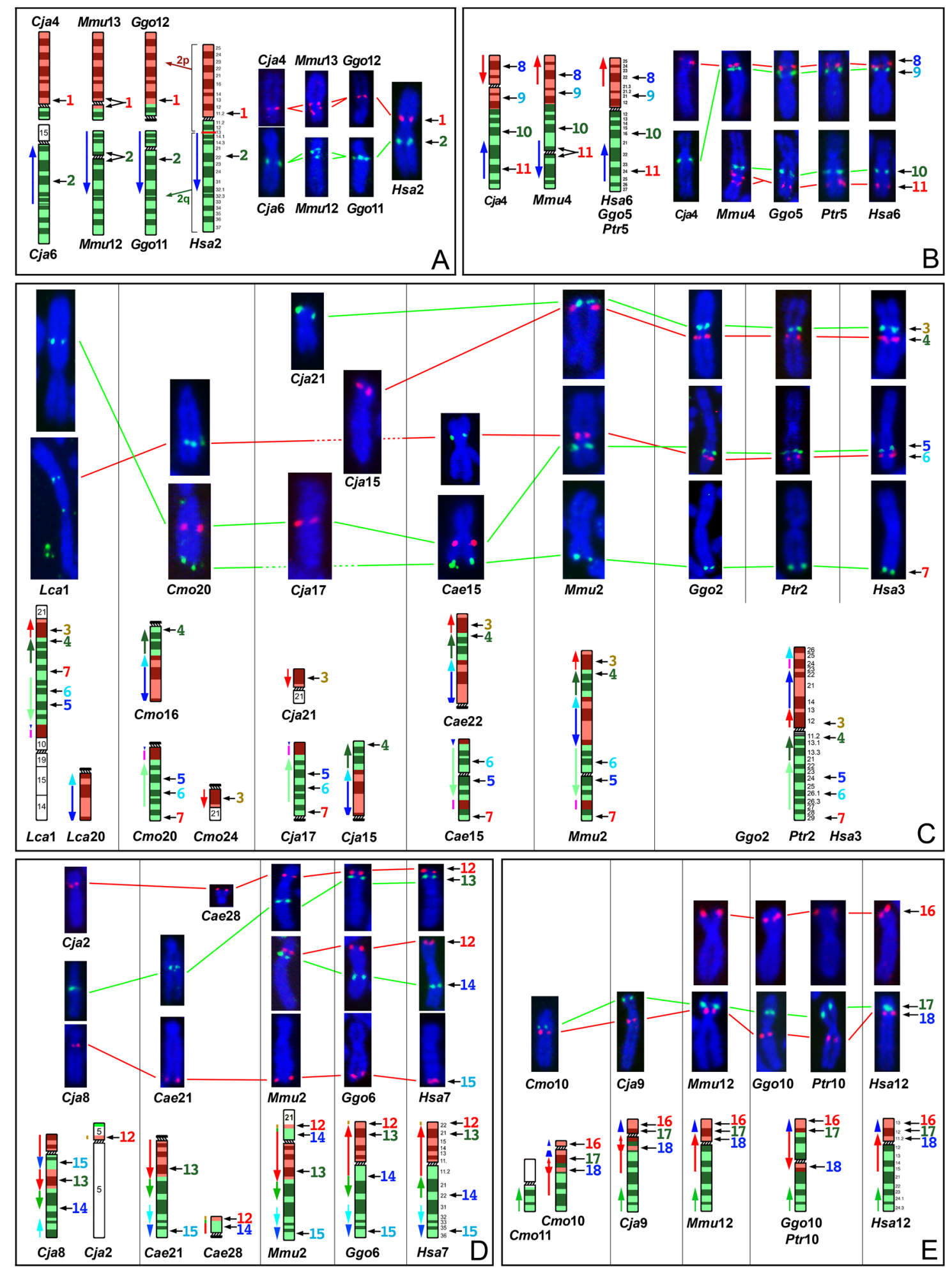

Figure 4. Location of human BAC probes on the primate metaphase chromosomes. The panels show the ideograms of the primate homologous chromosomes investigated and the relative FISH with the BAC probes (green and red signals); chromosomes were stained with DAPI (blue). Each panel shows human chromosomes Hsa2 (A), Hsa6 (B), Hsa3 (C), Hsa 7 (D), Hsal2 (E) and their homologues from G. gorilla (Ggo), P. troglodytes (Ptr), M. mulatta (Mmu), C. jacchus (Cja), C. moloch (Cmo), C. aethiops (Cae), and L. catta (Lca). The chromosomal bands, in the ideograms, of the human short and long arms (and the corresponding homologous chromosomal segments in the other primates) are stained in red and green, respectively. The arrows on the right of the chromosomes indicate the position of the BAC probes, numbered following the list in Table 1. The coloured arrows and lines on the left of the chromosomal ideograms indicate the evolutionary rearrangements. Red and green lines connect the same loci in the homologous chromosomes. 


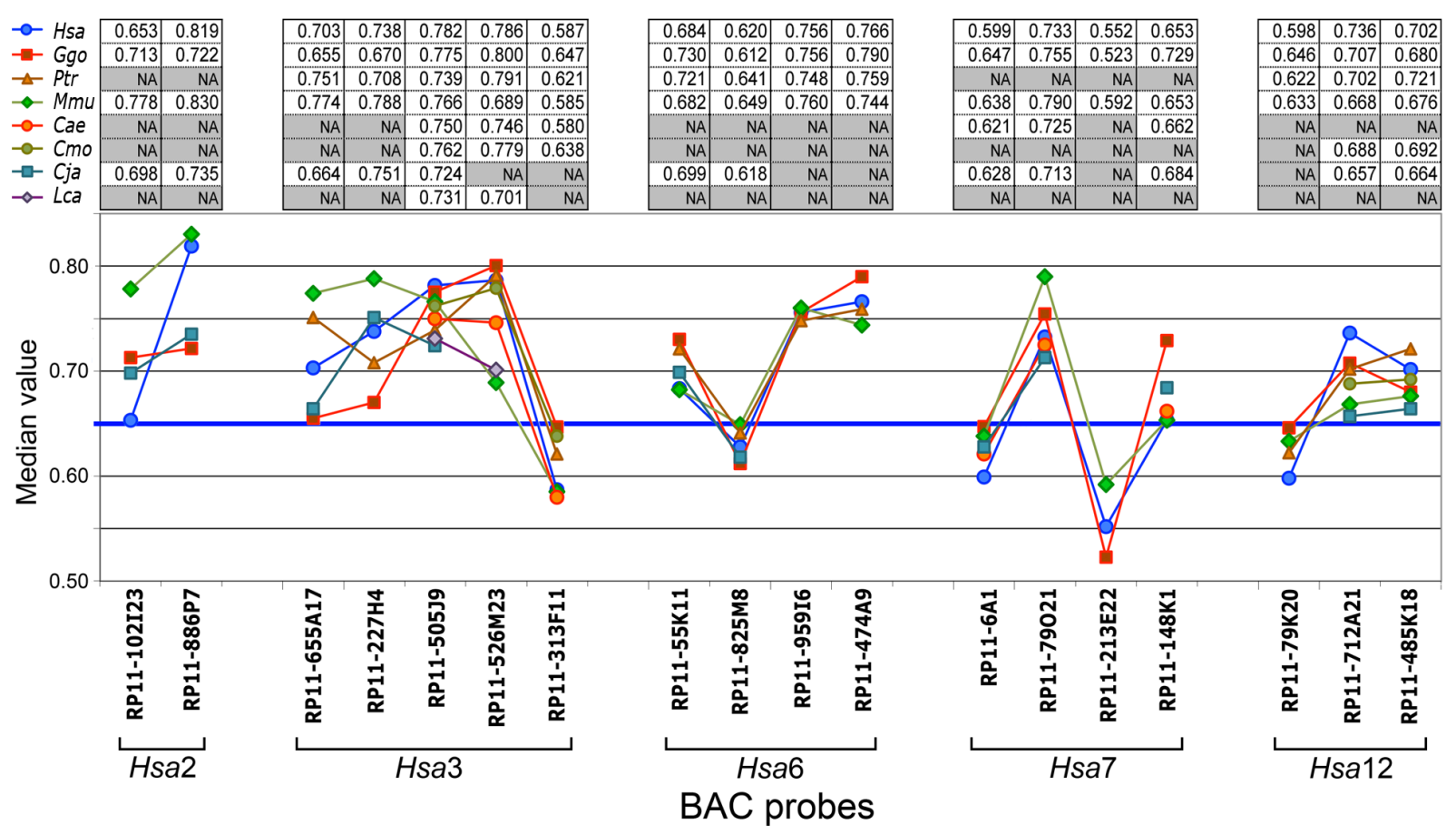

Figure 5. RNL of human BAC probes on primate cell nuclei. The RNL of the probes described in Table 1 are indicated by the relative median value (for simplicity of the graph visualisation, the confidence interval bars are not shown). The median values relative to probes hybridised on the human chromosomes are those shown in Figure 2. A coloured line connects, for each species, the median values of probes located in the same human chromosome: Hsa2, Hsa3, Hsa6, Hsa 7, and Hsa12, from left to right. Median values higher or lower than 0.65 are demarcated by the horizontal blue line. The symbols legend is shown in the upper left. The upper panel shows the BACs that worked for each species (with median value shown) and those that did not (NA: data not available). 




Figure 6. Compositional features of the human chromosomal bands with ENC, HCN, and AC sites. Upper and bottom left: GC-level of the chromosomal regions at a resolution of 850 bands (data from Costantini et al. 2007) corresponding to ENC, HCN and AC sites shown in Figure 1. Moreover, the GC-level of each band from the human chromosomes 19 and 22 are shown (bottom left). Bottom right: statistical evaluation of the GC-level (mean \pm I.C.) in the different types of chromosomal regions: $\mathrm{HCN}, \mathrm{ENC}, \mathrm{AC}$, chromosome 19 and chromosome 22. Represented in the upper part of the graph is the statistical correlation ( $p$-value obtained using the two-tailed t-test) between the indicated pair of data. 